\title{
Changes in Root Exudates' Composition and Their Ability to Release Cadmium Adhered to Soil in Four Lettuce Varieties under Cadmium Stress
}

\author{
Xiwang Tang ${ }^{1,2}$, Lili Yi ${ }^{1,2}$, Yu Song ${ }^{2}$, Xin $\mathrm{He}^{2}$, Lining Fang ${ }^{1,2 *}$, Jun’an Zhang ${ }^{1,2 * *}$ \\ ${ }^{1}$ Department of Environmental Science, Hebei University of Environmental Engineering, \\ Qinhuangdao 066102, China \\ ${ }^{2}$ Hebei Key Laboratory of Agroecological Safety, Hebei University of Environmental Engineering, \\ Qinhuangdao 066102, China
}

Received: 8 June 2020

Accepted: 7 August 2020

\begin{abstract}
To test the differences in root exudation under $\mathrm{Cd}$ stress among four lettuce varieties, a hydroponic culture experiment was conducted. In this study, four lettuce varieties were cultivated in Hoagland's solution containing $10 \mathrm{mg} \cdot \mathrm{L}^{-1} \mathrm{Cd}$ for 3 weeks. The water culture method was used to obtain a solution containing root exudates at the end of every week. In all four cultivars, the roots' secretion capacity increased over time, but the diversity of compounds in the root exudates and the soil-Cd activation capacity of root exudates decreased. The roots' secretion capacity was significantly negatively correlated with the soil-Cd activation capacity of the exudates $(\mathrm{r}=0.78, P<0.01)$ and with the number of compounds in root exudates $(\mathrm{r}=0.59, P<0.01)$. The soil-Cd activation capacity of root exudates was significantly positively correlated with the number of compounds in root exudates $(\mathrm{r}=0.69, P<0.01)$. Thus, under Cd stress, the lettuce root secretion capacity increased while the diversity of compounds in root exudates decreased, resulting in decreased soil-Cd activation.
\end{abstract}

Keywords: lettuce, cadmium stress, root exudates, soil-Cd activation capacity, 3D fluorescence spectrum scan

\section{Introduction}

In China, heavy metal pollution in soils is one of the most serious problems in crop cultivation, and it can cause serious economic losses [1-3]. When the growth environment is unfavorable or stressful, for

*e-mail: fanglining@hebuee.edu.cn

**e-mail: zhangjunan@hebuee.edu.cn example, because of heavy metal pollution, some plants can activate anti-stress responses to tolerate that environmental stress [4]. Cadmium (Cd) is a nonessential element for plants, and excessive $\mathrm{Cd}$ in soil adversely affects plant growth $[5,6]$. When the $\mathrm{Cd}$ concentration in soil exceeds a certain limit, plant growth is severely inhibited [7]. The symptoms of plants under $\mathrm{Cd}$ stress include dry and withered yellow leaves, short and thin roots, and fewer and undeveloped lateral roots. This greatly damages the quantity and quality of the crop [8]. 
Lettuce is a popular vegetable that is widely consumed worldwide. Unfortunately, it tends to absorb $\mathrm{Cd}$ from soil, leading to $\mathrm{Cd}$ enrichment in the edible parts. Thus, Cd-contaminated lettuce poses a risk to human health [1]. The $\mathrm{Cd}$ concentration in the leaves of lettuce grown in a substrate containing $\mathrm{CdCl}_{2}$ at $5 \mathrm{mg} \mathrm{kg}^{-1}$ was found to be 100 times the upper limit for vegetable products [9]. In general, lettuce shows high resistance to environmental stress, and grows well in soil with moderate $\mathrm{Cd}$ pollution. In previous studies, lettuces grown in soil spiked with Cd showed absolutely no symptoms such as dehydrated or yellow withering leaves [9-10]. Given that lettuce leaves containing high $\mathrm{Cd}$ concentrations could appear to be safe, eatable, and totally sellable, it is possible for Cd to gradually accumulate in the human body through the food chain. To prevent serious damage to human health, therefore, the lettuce $\mathrm{Cd}$ absorption process needs to be studied as a part of hazard control, and to devise appropriate food safety strategies.

Previous studies have shown that different lettuce varieties differ in their capability to absorb $\mathrm{Cd}$ from soil, even when they are grown under exactly the same conditions in the same Cd-polluted soil. There are many reasons for such differences, for example, differences among cultivars in migration of $\mathrm{Cd}$ from the soil to the root, and/or from the root to the shoot. In this study, we determined whether differences in root exudation among cultivars may contribute to differences in their $\mathrm{Cd}$ accumulation capacity. To this end, we analyzed three root exudate factors of different lettuce varieties under $\mathrm{Cd}$ stress: the total secretion amount, the number of compounds in secretions, and the soil-Cd activation capacity of secretions. We compared four Cd-accumulating varieties of lettuces cultivated in the same substrate. At the end of each week, young seedlings were carefully removed to a water-based hydroponic system to obtain root exudates. The highpurity water containing the exudates was analyzed to determine the number of compounds in the exudates, the total secretion amount, and the soil-Cd activating capacity. Then, to determine the contributions of the three root exudate factors to differences in $\mathrm{Cd}$ absorption among cultivars, the results were evaluated by correlation analyses.

\section{Experimental}

\section{Test Materials}

Four lettuce varieties were selected for these experiments: "Farthing bitter radicchio" (\#F), "Big oak no. 2" (\#O), "Giant American fast-growing lettuce" $(\# \mathrm{~A})$, and "Lisheng no. 2" (\#L). All lettuce seeds were purchased locally and were produced by Jingyan (China National Vegetable Engineering \& Technology Research Center). In previous studies, these four lettuce cultivars accumulated $\mathrm{Cd}$ in the above-ground parts to a concentration of $1.25 \pm 0.07(\# \mathrm{~F}), 1.55 \pm 0.12(\# \mathrm{O})$, $1.78 \pm 0.23(\# \mathrm{~A})$, and $1.90 \pm 0.44(\mathrm{HL}) \mathrm{mg} \mathrm{kg}^{-1} \mathrm{DW}$ when they were grown in a field with soil containing $\mathrm{Cd}$ at a concentration of $1.73 \mathrm{mg} \mathrm{kg}^{-1}$.

\section{Cultivation of Seedlings}

The seedlings were initially cultivated in a commercial nursery substrate comprising organic material (45\%), perlite, and vermiculite ( $\mathrm{pH}$ 5.5 6.5). The seedlings were cultivated in wooden trays $(60 \mathrm{~cm} \times 40 \mathrm{~cm} \times 20 \mathrm{~cm})$ filled with the substrate, and were watered using a sprinkler system. This kept the substrate at saturation conditions ideal for seedling growth. The seeds were evenly sprinkled over the surface of the substrate, and then covered with a thin layer of fine substrate powder. A mulching film was applied to keep the seeds moist and to provide protection. This film was removed after the emergence of small seedlings. The lettuce plants were watered using the sprinkler system during growth.

\section{Cadmium Stress Treatment}

Thirty-five days after seedling emergence, four robust single lettuce plants of the same size were selected for each variety, and were transferred from the seedling trays to hydroponic incubators $(40 \mathrm{~cm} \times$ $20 \mathrm{~cm} \times 15 \mathrm{~cm}$ opaque plastic boxes) for further growth in a water-based system, the hydroponic incubators was repeated 4 times. The seedlings were carefully measured and compared before being selected. Each seedling was then removed gently from soil and the nursery substrate particles adhering to the roots were gently shaken off. Once the root hairs were visible, the roots were rinsed 2 3 times with pure water to remove the smaller substrate fragments. The cleaned roots of the seedlings were then placed in Hoagland's complete culture solution (containing $10 \mathrm{mg} \mathrm{Cd} / \mathrm{L}$ in the form of $\mathrm{CdCl}_{2}$, $\mathrm{pH}=6$ ), with one variety per incubator. The solution in each container was covered with a foam board to protect the culture solution system from contamination. Each foam board had four evenly positioned holes, into which the seedlings were positioned. The seedlings were kept in place by a foam sealing method. Oxygen was pumped into the culture solutions by a compact air pump through four supply pipes, so that the roots of all cultivars were evenly supplied with oxygen. These hydroponic experiments were conducted in glass greenhouses at room temperature.

\section{Acquisition of Root Exudate Solution}

There are many different methods for collecting root exudates [11], including greenhouse collection methods (e.g. water culture collection, soil culture collection, substrate culture collection, and continuous root exudate collection) and field in situ collection methods (in situ extraction and monitoring). In this study, the 
lettuce root exudates were obtained by the water culture collection method as described by Lu et al. [12]. In this experiment, for each hydroponic incubator, one lettuce plant was temporarily removed from the culture solution for analysis three times: in week 1 (May $8^{\text {th }}$ ), week 2 (May $15^{\text {th }}$ ), and week 3 (May 22 ${ }^{\text {nd }}$ ). Each time, the roots were removed from the culture solutions and then immersed in deionized water for $5 \mathrm{~min}$. During this immersion period, the roots were gently shaken every $1 \mathrm{~min}$ to ensure that the culture solution was fully washed off. After that, each clean lettuce plant was placed into one light-shaded bottle containing $1 \mathrm{~L}$ high-purity water, and then kept in an illuminated incubator for 4 hours. The high-purity water culture solution containing the root exudates was collected for analysis. The plants were then put back into the hydroponic boxes, where the used culture solution had been replaced with fresh solution. The samples were stored at $4^{\circ} \mathrm{C}$ until analysis.

\section{Determination of Root Exudates Amount}

The burning oxidation-non-dispersive infrared absorption method were used to detect the total organic carbon (TOC) of the root exudate solution by a TOC-VCPH analyzer (Shimadzu, Kyoto, Japan). The TOC-VCPH is a PC controlled high-sensitivity analyzer that measures the amount of total organic carbon (TOC). The amount of organic matter in the water (secreted by plant roots) was calculated and used to determine each plant's root secretion capacity under Cd stress (mg TOC plant ${ }^{-1}$ hour $^{-1}$ ).

\section{Analysis of Substances in Root Exudates}

A FN4500 fluorescence spectrophotometer (Hitachi, Tokyo, Tokyo) was used to estimate the number of compounds in the root exudate solution. For each sample, 3D fluorescence spectra were produced from the scanning results. Fluorescence spectrum analysis is a simple, rapid, and efficient method to estimate the number of chemical species in complex mixtures such as plant root exudates [12]. The Hitachi FN4500 instrument had a $150 \mathrm{~W}$ xenon light as its light source, and was operated at $12,000 \mathrm{~nm}$ per min scanning speed, with a 5.0-nm excitation side slit, a 5.0-nm fluorescence side slit, a scanning range of $250 \sim 700 \mathrm{~nm}$ (excitation and emission), a 5-nm excitation wavelength interval, a 1-nm emission wavelength interval, and $400 \mathrm{~V}$ photomultiplier tube voltage. The "spectrum supplement" status was switched on, and the response was automatic.

\section{Evaluation of Soil Cd Activation Capacity of Root Exudates}

To measure the capacity of root exudates to activate $\mathrm{Cd}$ bound to soil, each root exudate sample was mixed with Cd-contaminated soil $\left(1 \mathrm{~g} \mathrm{~kg}^{-1}, \mathrm{pH}=7.3\right)$ and oscillated to leach $\mathrm{Cd}$ (soil to water ratio, 2:1) for $2 \mathrm{~h}$ at $25^{\circ} \mathrm{C}$. The solution was filtered and then analyzed using a flame graphite furnace atomic spectrophotometer (Hitachi Z2000) to determine the Cd concentration , using the diethylenetriaminepentaacetic acid extraction method as a reference [3]. The Cd-contaminated soil was prepared in April 2013 and comprised Lou soil mixed with a solution of $\mathrm{CdCl}_{2}$. After mixing, the Cd-contaminated soil was naturally air-dried and then passed through a 100-mesh sieve. The Cd-contaminated soil was then sealed and stored in a glass jar until use.

\section{Data Analysis}

Data were processed using Excel 2010 and Sigmaplot 12.5. One-way ANOVA and Fisher's LSD test method were used to detect and compare differences among treatments. Pearson's method was used for the correlation analyses among root exudate TOC contents, number of peak points in root exudate threedimensional (3-D) fluorescence scans, and root exudate soil-Cd activation capacity.

\section{Results and Discussion}

Results

\section{Changes of Total Amount of Lettuce Root Exudates under Cd Stress}

The total amount of root exudates from the four lettuce varieties under $\mathrm{Cd}$ stress is shown in Fig. 1 ( $n=3, \alpha=0.05$ ). The figure shows three groups of results, from week 1 (solution samples collected on May $8^{\text {th }}$ ), week 2 (samples collected on May $15^{\text {th }}$ ) and week 3 (samples collected on May $22^{\text {nd }}$ ). In week 1 , there was no significant difference in the amount of root exudates among the four lettuce varieties. However, in week 2, a larger amount of root exudates was produced by \#A than by the other three varieties. For varieties $\# \mathrm{O}, \# \mathrm{~F}$, and $\# \mathrm{~L}$, the total amount of root exudates was markedly higher in weeks 2 and 3 than in week 1, and higher in week 3 than in week 2 . In contrast, variety \#A, which secreted more exudates than the other three varieties in week 2 , secreted a smaller amount of exudates in week 3 than in week 2. In week 3 , \#O secreted the largest amount of exudates.

Notably, the total amount of root exudates generally increased over time in all four varieties, except $f$ or \#A. In \#A, the total amount of root exudates from a single plant was 6.26 times higher in week 2 (2.23 mg plant ${ }^{-1}$ hour $\left.^{-1}\right)$ than in week $1\left(0.27 \mathrm{mg} \mathrm{plant}^{-1}\right.$ hour $^{-1}$ ), but slightly decreased in week 3. In $\# \mathrm{O}, \# \mathrm{~F}$, and $\# \mathrm{~L}$, the amount of root exudates of increased gradually from week 1 to week 3 , showing a consistent and simpler pattern, with \#O showing an increase from 0.14 to $2.47 \mathrm{mg}$ plant $^{-1}$ hour $^{-1}$ and \#L showing 


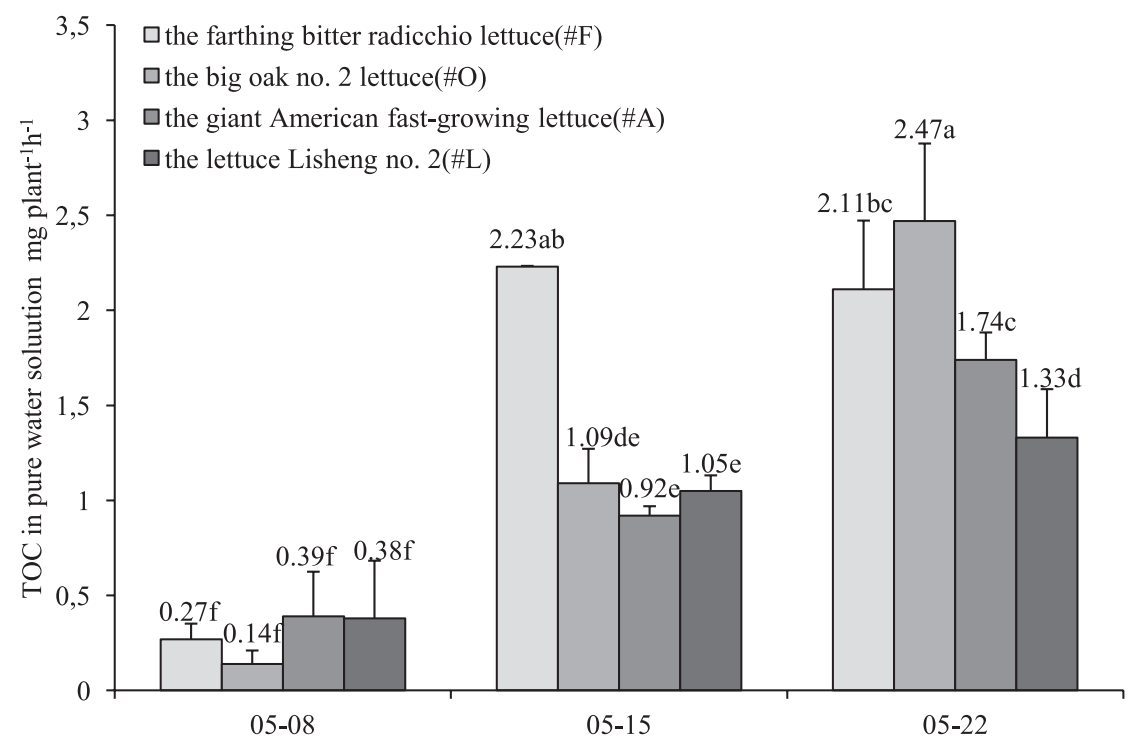

Fig. 1. The TOC concentration of the root exudates solution $(n=3, \alpha=0.05)$.

a relatively moderate change from 0.38 to $1.33 \mathrm{mg}$ plant $^{-1}$ hour ${ }^{-1}$.

\section{Changes in Diversity of Compounds in Root Exudates of Lettuce under Cd Stress}

The amount and types of chemical species in the lettuce root exudates in secretion solutions were determined by 3D fluorescence spectra characterization. As shown in Fig. 2, these analyses were conducted in weeks 1,2 , and 3 for the four varieties. Comparing the $3 \mathrm{D}$-scanning contour maps among the four varieties in week $1(1-\# \mathrm{~A}, 1-\# \mathrm{O}, 1-\mathrm{HF}$, and 1-\#L) the peak ranges were similar for the four different varieties, indicating that the organic species in the four varieties were largely the same in week 1 . The 3D scanning contour maps were also similar among the four varieties in weeks 2 and 3.

Comparing the 3D scanning contour maps for each variety among the three time points, all four varieties showed diminishing peak ranges from week 1 to week 3 , indicating that the diversity of organic substances in the root exudates decreased from week 1 to week 2 , and from week 2 to week 3 .
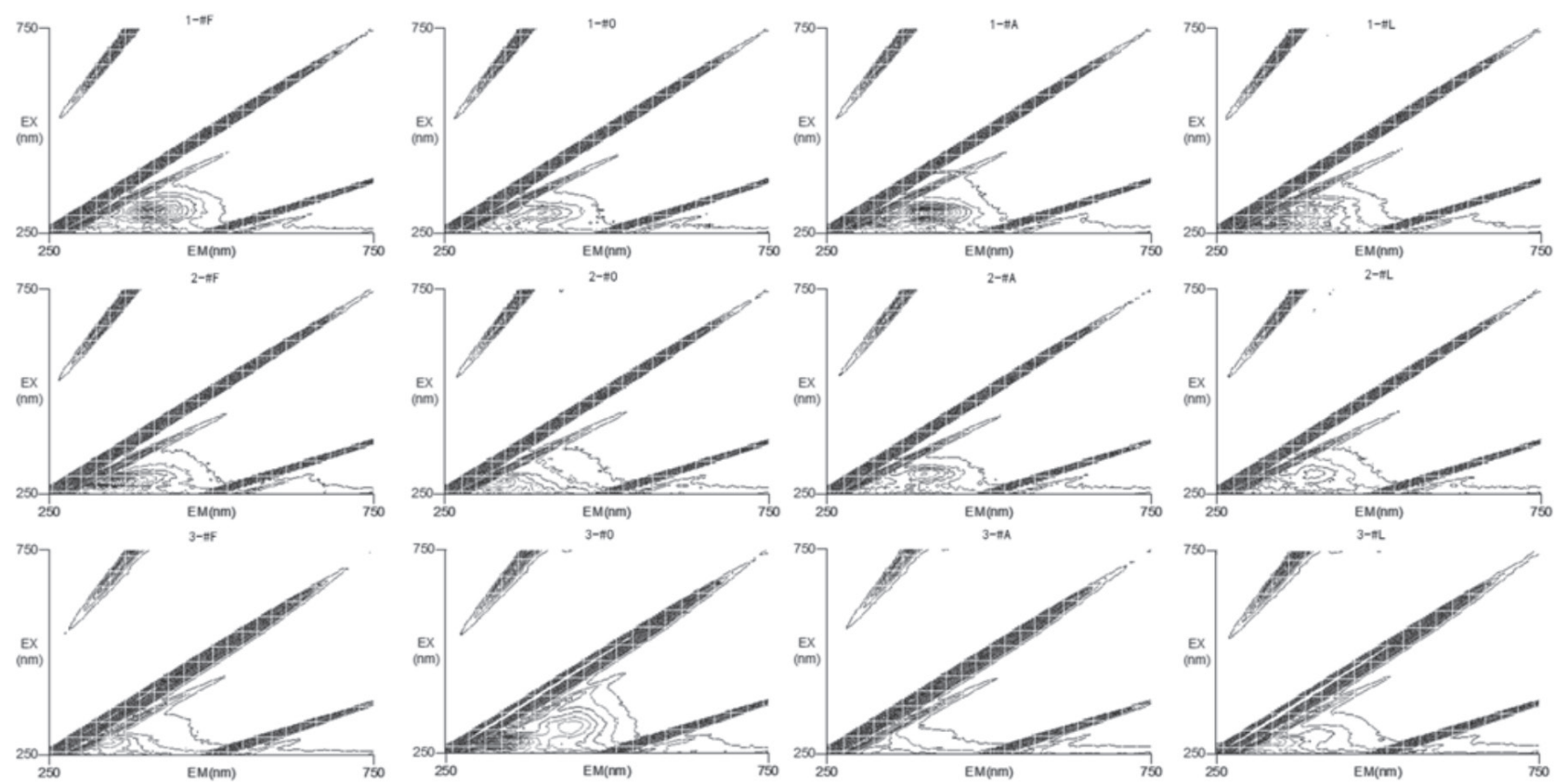

Fig. 2. 3D-scanning contour maps of root exudate solutions.Note: Numbers $1,2,3$ before letter represent May $8^{\text {th }}, 15^{\text {th }}$, and $22^{\text {nd }}$, respectively. Four lettuce varieties are: "Farthing bitter radicchio" (\#F), "Big oak no. 2" (\#O), "Giant American fast-growing lettuce" (\#A), and "Lisheng no. 2" (\#L). 


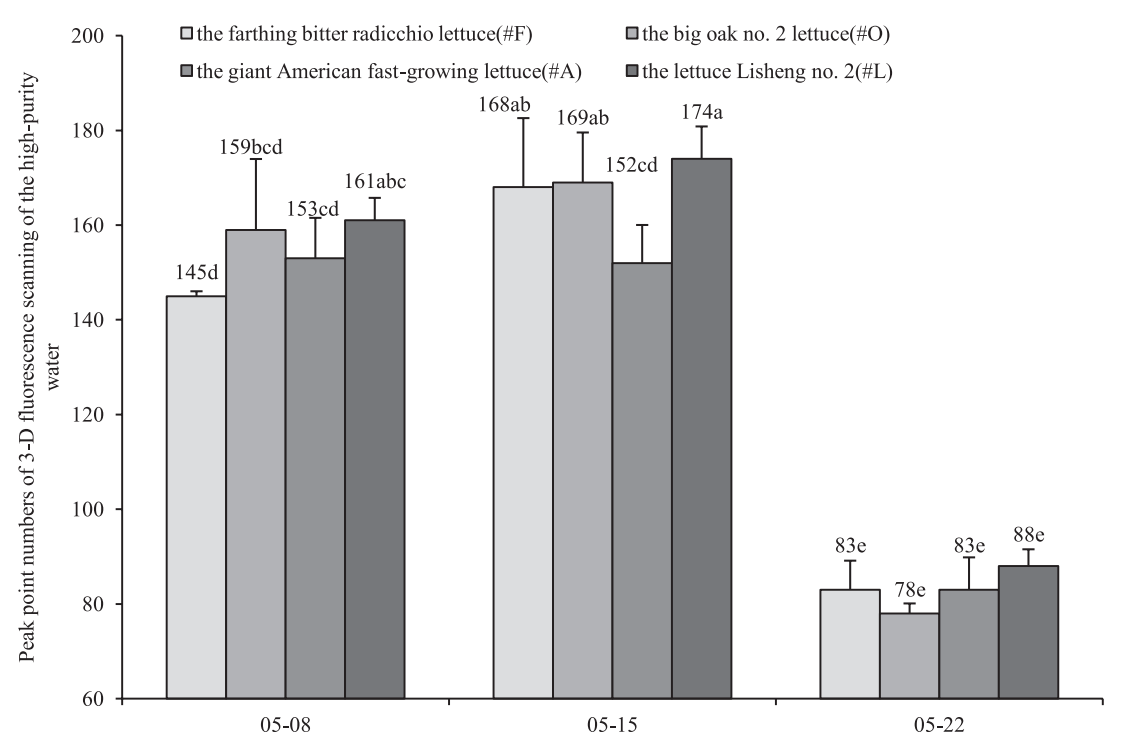

Fig. 3. The peak point numbers of 3-D fluorescence scanning of the root exudates solutions in May $8^{\text {th }}, 15^{\text {th }}, 22^{\text {nd }}(\mathrm{n}=3, \alpha=0.05)$.

To further analyze the root secretions, the number of fluorescence peaks in the 3D fluorescence spectra was counted [13-14]. As shown in Fig. 3, as many as 145 fluorescence peaks were detected for \#A in week 1 , indicative of a diverse range of substances in the root exudate. There were even more peaks in the spectra of $\# \mathrm{O}, \# \mathrm{~F}$, and $\# \mathrm{~L}(159,153$, and 161 peaks, respectively). Given that a certain organic substance can correspond to more than one peak, this value does not represent the exact number of substances in the root exudates. However, the number of peaks detected in a 3D fluorescence spectrum is significantly positively correlated with the number of compounds in the mixture. This helps to explain the moderately increased peak numbers for all four varieties in week 2 (168 peaks for \#A, 169 peaks for \#O, 152 peaks for $\# \mathrm{~F}$, and 174 peaks for $\# \mathrm{~L})$. In $\# \mathrm{~A}$, there were 23 more peaks in week 2 than in week 1 . In week 3 , there were fewer peaks in the fluorescence spectra: 83 peaks (\#A), 78 peaks $(\# \mathrm{O}), 83$ peaks $(\# \mathrm{~F})$, and 88 peaks $(\# \mathrm{~L})$. This was indicative of a less diverse range of compounds in the root exudates in the third week of Cd stress. In summary, the results of the 3D scanning contour map and 3D fluorescence spectrum peak analyses indicated that the number of compounds in the root exudates decreased over time in all four lettuce cultivars under Cd stress.

\section{Changes in Soil-Cd Activation Capacity of Root Exudates}

We tested the ability of root exudates to activate soilbound $\mathrm{Cd}$ by using the culture solutions to extract $\mathrm{Cd}$ from artificially contaminated soil. For these analyses,

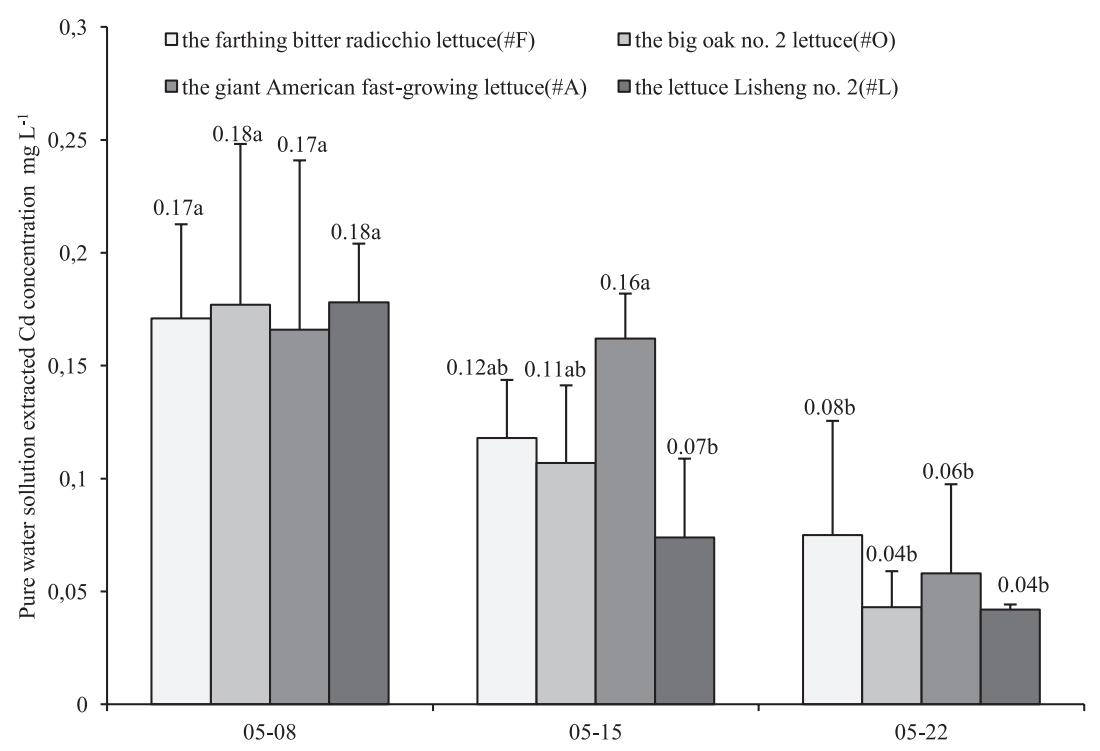

Fig. 4. The Cd concentration in root exudates high pure water solution after extracted the Cd polluted soil $(\mathrm{n}=3, \alpha=0.05)$. 
we prepared $\mathrm{Cd}$-contaminated soil by adding a $\mathrm{CdCl}_{2}$ solution to soil, and then used the secretion solutions to extract the $\mathrm{Cd}$ from the soil particles. As shown in Fig. 4, in week 1, there was no significant difference in the amount of $\mathrm{Cd}$ extracted from soil by secretion solutions from the four lettuce varieties (\#A, $0.17 \mathrm{mg} / \mathrm{L}$; \#O, $0.18 \mathrm{mg} / \mathrm{L}$; \#F, $0.17 \mathrm{mg} / \mathrm{L}$; \#L, $0.18 \mathrm{mg} / \mathrm{L}$ ). By week 3 , these values had decreased significantly $(P<0.05)$ for all four varieties (\#A, $0.08 \mathrm{mg} / \mathrm{L}$; \#O, $0.04 \mathrm{mg} / \mathrm{L}$; \#F, $0.06 \mathrm{mg} / \mathrm{L}$; \#L, $0.04 \mathrm{mg} / \mathrm{L})$. Therefore, with increasing cultivation time under Cd stress, the ability of secretion solutions to release water-soluble $\mathrm{Cd}$ from soil particles decreased.

\section{Correlation Analysis}

We determined lettuce roots' secretion capacity (mg TOC liter ${ }^{-1}$ hour $^{-1}$ ), and the number of peak points in the 3-D fluorescence scans of water culture solutions (an indicator of the number of organic compounds in root exudates). The concentration of $\mathrm{Cd}$ leached from Cd-contaminated soil $(\mathrm{mg} / \mathrm{L})$ by the water culture solution represented the lettuce root exudates' soil-Cd activation capacity. Correlation analyses between pairs of these three factors were conducted using Pearson's method and the results are shown in Table 1.

As shown in Table 1, the lettuce root exudates' soil-Cd activation capacity was significantly negatively correlated $(\mathrm{r}=0.78, \mathrm{P}<0.01)$ with the roots' secretion capacity. The root exudate soil-Cd activation capacity was highly positively correlated $(r=0.69$, $P<0.01$ ) with number of organic compounds in root secretions. There was a highly significant negative correlation between the lettuce roots' secretion capacity and the number of organic species in root exudates $(\mathrm{r}=-0.59, P<0.01)$.

\section{Discussion}

In this study, the four lettuce varieties showed significant differences in $\mathrm{Cd}$ absorption capacity at the early stage of $\mathrm{Cd}$ stress. However, across the whole experimental period, there were no significant differences among the four cultivars in the total amount of root exudates, number of organic species in root exudates, or the soil-Cd activation capacity of root exudates. Therefore, the differences in $\mathrm{Cd}$ absorption

Table 1. Pearson's correlation analyses between lettuce roots' secretion capacity (TOC content) (A), number of peak peaks in 3-D scans (B), and root exudates' soil-Cd activation capacity (C).

\begin{tabular}{|c|c|c|c|}
\hline & $A$ & $B$ & $C$ \\
\hline $\mathrm{A}$ & 1 & & \\
\hline $\mathrm{B}$ & $-0.78^{* *}$ & 1 & \\
\hline $\mathrm{C}$ & $-0.59^{* *}$ & $0.69^{* *}$ & 1 \\
\hline
\end{tabular}

Note: ** highly significant, $\alpha=0.01$ among these four lettuce varieties are probably due to other factors. Despite this finding, we obtained some interesting results in these experiments.

Viscose, exogenous enzymes, organic acids, sugars, phenols, and various amino acids are all typical organic substances in root exudates $[15,16]$. As suggested by Chen et al. [17], under nutrient and environmental stress, the proportions of viscose, enzymes, and organic acids in root secretions may change as plants adapt to changes in their environment. In this study, the longer the lettuce plants were grown under Cd stress in the hydroponic system, the larger the total amount of root exudates in term of TOC content. However, in all four lettuce varieties, the diversity of organic compounds in root exudates decreased over time. This is consistent with the results of other studies on the responses of roots to Cd stress [18-21]. Qiang et al. showed that the secretion of polypeptides, amino acids, and amides from soybean roots increased under Cd stress [20]. Qin et al.found that the mass concentration of total organic acids, free amino acids, and soluble sugar species in root exudates of Sonchus asper L. Hill increased markedly under Cd stress [21]. Zhang et al. analyzed the root secretions of wheat under Cd stress, and detected decreasing concentrations of secondary metabolites and amino acids, but increasing production of electrolytes and sugars [19].

Many studies have shown that various chemicals in plant root exudates can affect soil Cd bioavailability [6, 22-24]. Wu reported that fresh maize root exudates could inhibit soil $\mathrm{Cd}$ adsorption [24] and Lin et al. reported that water-soluble exudates from wheat roots increased $\mathrm{Cd}$ adsorption in red soil [22]. In this study, with prolonged cultivation in the Cd-contaminated hydroponic culture system, the lettuce root secretion capacity increased, but the diversity of compounds in the exudates decreased and the soil-Cd activation capacity of root exudates also decreased. One explanation for this is that as the plants grew, they produced more total root exudates as the plant size increased, but the percentage of low molecular weight organic acids in root exudates decreased (see Fig. 2). Root exudates contain two major classes of compounds: low molecular weight compounds including amino acids, organic acids, phenolics, and sugars; and high molecular-weight compounds including mucilage and proteins [17]. In general, low molecular weight organic acids can decrease soil $\mathrm{Cd}$ adsorption. Chen et al. studied the ability of three types of root exudates (citric acid, glycine, and maltose) to activate $\mathrm{Cd}$ ions in soils, and found that they could rank, from strongest ability to weakest, as follows: citric acid $>$ glycine $>$ maltose. Tao et al. found that low molecular weight organic acids such as tartaric acid could significantly increase the solubility of $\mathrm{Cd}$ minerals [6]. The decreased amounts of low molecular weight organic acids in lettuce root exudates over time could explain the decrease in the soil-Cd activation capacity. This suggests that one of the tolerance mechanisms of lettuce 
plants under Cd stress is to decrease the amount of low molecular weight compounds in root exudates, leading to lower activation of soil- $\mathrm{Cd}$ in the soil environment. This would effectively reduce the uptake of soil-Cd by roots.

\section{Conclusion}

There was no significant difference in the total amount of root exudates, diversity of compounds in root exudates, and soil-Cd activation capacity among four different lettuce varieties under $\mathrm{Cd}$ stress. However, all of these factors changed over time during 3 weeks of growth under $\mathrm{Cd}$ stress. For lettuces cultivated in a hydroponic system under Cd stress, the amount of root exudates was significantly negatively correlated with both the number of different compounds in root exudates and the soil-Cd activation capacity of root exudates. The number of compounds in root exudates was significantly positively correlated with the soil-Cd activation capacity. As the duration of cultivation under $\mathrm{Cd}$ stress extended, the root exudates of the four cultivars contained fewer organic compounds. This is one possible reason for the decrease in soil-Cd activation capacity of root exudates over time.

\section{Acknowledgements}

This research was sponsored by Natural Science Foundation of Hebei Province of China (grant No. D2019415034), Program of Key research and development program of Hebei province (grant No. 18222906D), Program for Chinese Scholars with Overseas Studying Backgrounds in Hebei (No. C201823), and Doctoral Starting-up Foundation of Hebei University of Environmental Engineering (grant No. BJ201801). We thank Jennifer Smith, PhD, from Liwen Bianji, Edanz Group China (www.liwenbianji. $\mathrm{cn} / \mathrm{ac}$ ), for editing the English text of a draft of this manuscript.

\section{Conflict of Interest}

The authors declare no conflict of interest.

\section{References}

1. TANG X., PANG Y., JI P., GAO P., NGUYEN T.H., TONG Y. Cadmium uptake in above-ground parts of lettuce (Lactuca sativa L.). Ecotox Environ Safe, 125 (125), 102, 2016.

2. DU J., NA L., YONG-NING W., QI-XING Z. Variation in accumulation of heavy metals in vegetables and low accumulation vegetable varieties:a review. Journal of Agro-Environment Science, 38 (06), 1193, 2019.
3. TANG X., SONG Y., HE X., YI L. Enhancing phytoremediation efficiency using regulated deficit irrigation. Pol J Environ Stud, 28 (4), 2399, 2019.

4. FAROOQ M., WAHID A., KOBAYASHI N., FUJITA D., Basra S.M.A. Plant drought stress: Effects, mechanisms and management. Agronomy for Sustainable Development, 29 (1), 185, 2009.

5. DONG J., MAO W.H., ZHANG G.P., WU F.B., CAI Y. Root excretion and plant tolerance to cadmium toxicity - a review. Plant Soil and Environment, 53 (5), 193, 2007.

6. CHEN C., LI Z., LI S., DENG N., MEI P. Effects of root exudates on the activation and remediation of cadmium ion in contaminated soils. Environmental Science and Pollution Research, 27 (3), 2926, 2020.

7. SARVAJEET SINGH G., NARENDRA T. Cadmium stress tolerance in crop plants: Probing the role of sulfur. Plant signaling \& behavior, 6 (2), 215, 2011.

8. LARS J., ÅKESSON A. Current status of cadmium as an environmental health problem. Toxicology and Applied Pharmacology, 238 (3), 201, 2009.

9. BERTHOMIEU C., LETTUCE P. (Lactuca sativa): A species with a high capacity for cadmium (Cd) accumulation and growth stimulation in the presence of low $\mathrm{Cd}$ concentrations. The Journal of Horticultural Science and Biotechnology, 88 (6), 783, 2013.

10. PEREIRA B., ROZANE D.E., ARAUJO S.R., BARTH G., QUEIROZ R., NOGUEIRA T., MORAES M.F., CABRAL C.P., BOARETTO A.E., MALAVOLTA E. Cadmium availability and accumulation by lettuce and rice. Revista Brasileira de Ciência do Solo, 35 (2), 645, 2011.

11. GUO W., ZHANG Z., LIU Q., YIN H. Research progress of root exudates collection technology. Yingyong Shengtai Xuebao, 30 (11), 3951, 2019.

12. LU S., HU H., SUN Y., YANG J. Study on the growth characteristics and root exudates of three wetlands plants at different culture conditions. Environmental Science, 30 (0), 1901, 2009.

13. JUN S., ZHI-GANG W., KE F. Characterization techniques of dissolved organic pollutants in wastewater by ThreeDimensional fluorescent spectroscopy and its application in environmental analysis. Journal of Atmospheric and Environmental Optic,. 6 (4), 243, 2011.

14. SHIYU C., YAN L., AIMIN L. Application of threedimensional fluorescence spectroscopy in the study of dissolved organic matter. ENVIRON SCI TECHNOL, $\mathbf{3 8}$ (05), 64, 2015.

15. PREECE C., PENUELAS J. A return to the wild: Root exudates and food security. TRENDS PLANT SCI, 25 (1), 14-21, 2020.

16. VIVES-PERIS V., DE OLLAS C., GOMEZ-CADENAS A., PEREZ-CLEMENTE R.M. Root exudates: From plant to rhizosphere and beyond. PLANT CELL REP. 39 (0), 3-17, 2019.

17. CHEN Y., WANG Y., YEH K. Role of root exudates in metal acquisition and tolerance. Current Opinion in Plant Biology, 39 (0), 66, 2017.

18. TU S., SUN J., GUO Z., GU F. On relatiosnship between root exudates and plant nutrition in rhizosphere. Ecology and Environmental Sciences, 9 (01), 64, 2000.

19. ZHANG L., WANG H. Changes of Root Exudates to Cadmium Stress in Wheat (Triticum aestivm L.). Acta Ecologica Sinica, 22 (04),496, 2002.

20. QIANG W., CHEN T., TANG H., FENG H., LIZHE A. Effect of cadmium and enhanced uv-b radiation on soybean root excretion. Acta Phytoecologica Sinica, 27 (03), 293, 2003. 
21. QIN L., LI Y., ZU Y., HE Y., WANG J., CHEN J. Effects of $\mathrm{Cd}$ Contamination on the root exudates of Sonchus asper L. Hill. Ecology and Environmental Sciences, 21 (03), 540, 2012.

22. LIN Q., CHEN Y., CHEN H., ZHENG C. Study on chemical behavior of root exudates with heavy metals. Plant Nutrition and Fertilizing Science. 9 (04), 425, 2003.
23. TAO Q., ZHAO J., LI J., LIU Y., LUO J., YUAN S., LI B., LI Q., XU Q., YU X., HUANG H., LI T., WANG C. Unique root exudate tartaric acid enhanced cadmium mobilization and uptake in Cd-hyperaccumulator Sedum alfredii. J HAZARD MATER. 383 (0), 121177, 2020.

24. WU Q. Effects of root exudates on bioavailability of cadmium. Soils, 25 (05), 257, 1993. 